\title{
Hypertension and the development of hearing loss
}

\author{
Kensuke Toyama ${ }^{1} \cdot$ Masaki Mogi ${ }^{1}$
}

Received: 15 September 2021 / Accepted: 23 September 2021 / Published online: 27 October 2021

(c) The Japanese Society of Hypertension 2021

The World Health Organization cautions that over $5 \%$ of the world's population (432 million adults and 34 million children) may require rehabilitation to address 'disabling' hearing loss and estimates that over 700 million people will be suffering from hearing loss by 2050 [1]. Hearing loss, a common condition in older adults, is not simply a problem of "not hearing well". A loss of hearing ability inhibits communication and social interactions, resulting in social isolation [2]. Hearing loss was recently described as the greatest risk factor for dementia [3], suggesting that social isolation and dementia could be cross-linked through hearing impairment. Thus, hearing maintenance is an important factor for preserving quality of life in older people.

Aging, heredity, noise exposure, and drug-induced ototoxic side effects are well known as the main causes of hearing loss [4], but little is known about other key risk factors for hearing impairment. Epidemiologic studies support significant links between hearing-loss development and vascular risk factors such as dyslipidemia and diabetes [5, 6]. Furthermore, recent studies have noted that low or declining platelet levels are also important and novel risk factors for the development of hearing loss [7]. Managing these risk factors is crucial to prevent the development of hearing impairment, since no effective drug therapies are approved that are capable of protecting or restoring hearing, and treatment for hearing loss is limited to clinical devices [4].

The effects of hypertension on the development of hearing impairment to date have primarily been noted in cross-sectional studies [5]. However, the current investigation by Miyata et al. features several strengths, including a large number of participants, a longitudinal design, and the use of facility-based measured blood

Kensuke Toyama

one.of.ken@icloud.com

1 Department of Pharmacology, Ehime University Graduate School of Medicine, Toon, Japan pressures and audiometry test results (not self-reported data) [8]. The authors excellently demonstrate that high blood pressure is a significant risk factor for the development of hearing impairment [8].

The mechanisms underlying the association between hypertension and hearing-loss development are not yet fully understood. Vascular injury might be involved, as it can reduce capillary blood flow, resulting in reduced oxygen transport and tissue hypoxia, thus causing hearing loss [9]. In addition, a blood-labyrinth barrier (BLB) exists in the inner ear, similar to the blood-brain barrier in the brain, separating the vasculature and the inner ear fluids (endolymph and perilymph) [4]. Blood supply to the cochlea is critically important for sustaining the production of endolymph, and the BLB is instrumental in maintaining inner ear fluid ionic homeostasis [10]. If the BLB of the stria vascularis in the cochlea becomes dysfunctional (e.g., through a disruption of tight junctions) via hypertension-induced vascular injury, this could disrupt ionic homeostasis in the endolymph, resulting in hair cell depolarization loss and hearing loss (Fig. 1).

Given that the data indicate that hypertension accelerates hearing disability, interventions to control hypertension could become novel strategies to protect against or treat hearing impairment. However, to the best of our knowledge, there are no clinical guidelines describing how best to manage hypertensive hearing-loss patients. The evidence linking hearing loss and hypertension seems to be conclusive, but further prospective studies are required to clarify appropriate therapeutic strategies for hearing loss independent of device aids. Furthermore, the molecular mechanisms connecting hypertension and hearing-loss development still require investigation. 


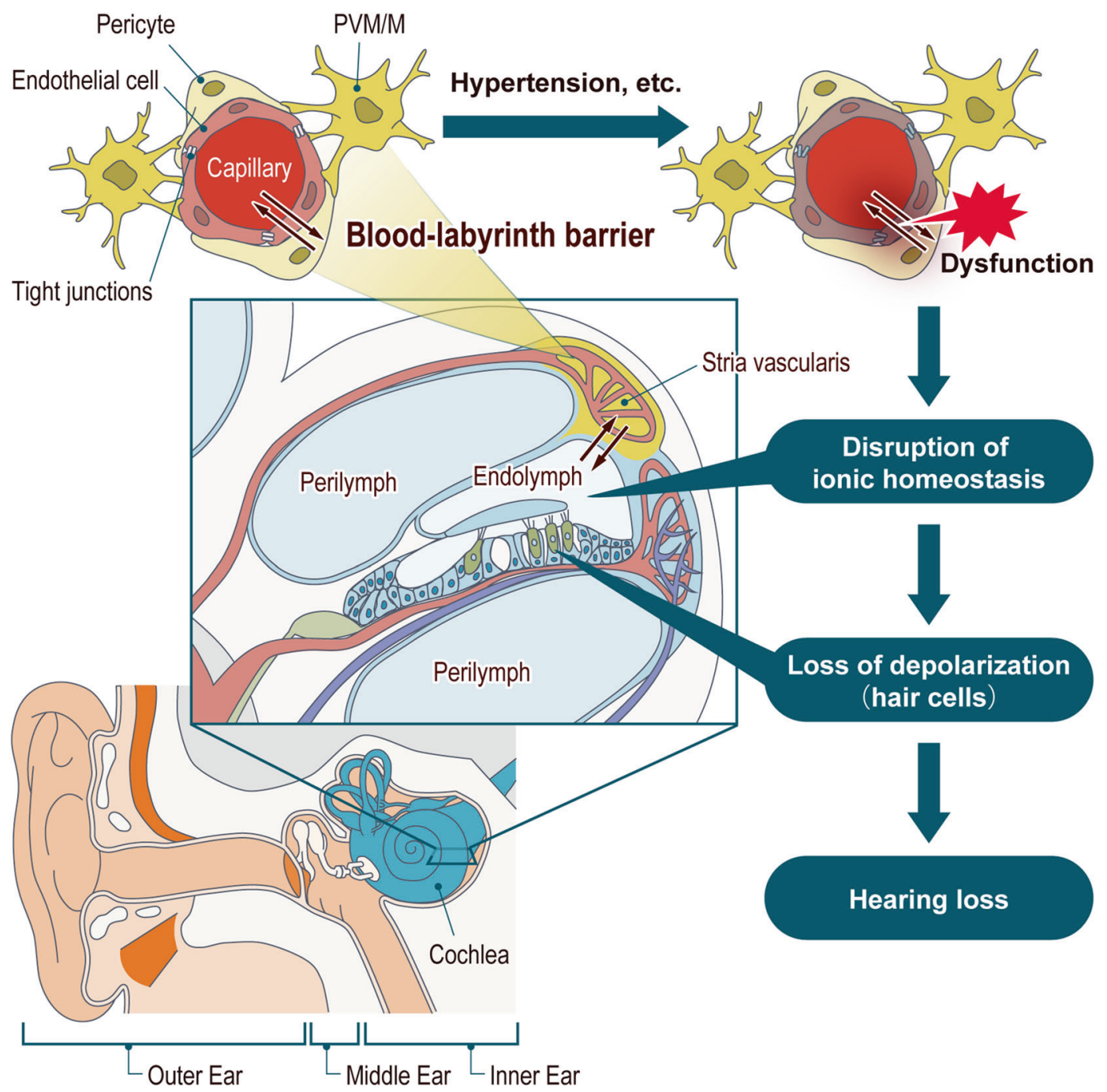

Fig. 1 One of the possible mechanisms between hypertension and hearing loss development via blood-labyrinth barrier dysfunction. PVM/M perivascular-resident macrophage-like melanocytes

\section{Compliance with ethical standards}

Conflict of interest The authors declare no competing interests.

Publisher's note Springer Nature remains neutral with regard to jurisdictional claims in published maps and institutional affiliations.

\section{References}

1. World Health Organization (Home/Newsroom/Fact sheets/Detail/ Deafness and hearing loss). https://wwwwhoint/news-room/factsheets/detail/deafness-and-hearing-loss.

2. Shukla A, Harper M, Pedersen E, Goman A, Suen JJ, Price C, et al. Hearing Loss, Loneliness, and Social Isolation: a Systematic Review. Otolaryngol Head Neck Surg. 2020;162:622-33.

3. Livingston G, Huntley J, Sommerlad A, Ames D, Ballard C, Banerjee S, et al. Dementia prevention, intervention, and care: 2020 report of the Lancet Commission. Lancet. 2020;396:413-46.
4. Nyberg S, Abbott NJ, Shi X, Steyger PS, Dabdoub A. Delivery of therapeutics to the inner ear: the challenge of the blood-labyrinth barrier. Sci Transl Med. 2019;11:eaao0935.

5. Hara K, Okada M, Takagi D, Tanaka K, Senba H, Teraoka M, et al. Association between hypertension, dyslipidemia, and diabetes and prevalence of hearing impairment in Japan. Hypertens Res. 2020;43:963-8.

6. Bainbridge KE, Hoffman HJ, Cowie CC. Risk factors for hearing impairment among U.S. adults with diabetes: National Health and Nutrition Examination Survey 1999-2004. Diabetes Care. 2011;34:1540-5.

7. Abe Y, Toyama K, Kazurayama M, Tanaka S, Yamaizumi M, Ueno M, et al. Low-Normal Platelets and Decreasing Platelets Are Risk Factors for Hearing Impairment Development. Laryngoscope. 2021;131:E1287-E1295.

8. Miyata J, Umesawa M, Yoshioka T, Iso H. Association between high systolic blood pressure and objective hearing impairment among Japanese adults: a facility-based retrospective cohort study. Hypertens Res. 2021 (in press). https://doi.org/10.1038/s41440021-00737-8. 
9. Agarwal S, Mishra A, Jagade M, Kasbekar V, Nagle SK. Effects of hypertension on hearing. Indian J Otolaryngol Head Neck Surg. 2013;65:614-8. Suppl 3
10. Trune DR. Ion homeostasis in the ear: mechanisms, maladies, and management. Curr Opin Otolaryngol Head Neck Surg. 2010;18:413-9. 